\title{
REPRESENTATION OF NONEQUILIBRIUM THERMODYNAMIC SYSTEM BY TWO-COMPONENT SUPERFIELD
}

\author{
A. I. Olemskoi, I. V. Koplyk, V. A. Brazhnyi \\ Sumy State University, Ukraine, Sumy, UA-244007, 2 Rimskii-Korsakov Str. \\ E-mail: postmaster@sgu.sumy.ua
}

(Received July 16, 1996)

\begin{abstract}
For the thermodynamical system described by the Langevin equation with the additive white noise the expression for a generating potential as a dependence on a two-component superfield is determined. The usual component of this superfield represents an order parameter, and the Grassmannian one represents a selfconsistent field conjugated by this parameter. Within the first order perturbation theory over particle interaction and unharmonicity the explicit expressions for wave-frequency dependence of autocorrelators of the real order parameter and the corresponding conjugated field are determined. The condition of stability breaking and the characteristic system scale are found. For the complex order parameter a form of frequency dependence of the corresponding correlators is determined numerically for different parameters of the theory.

Key words: order parameter, conjugated field, superfield, correlator.
\end{abstract}

PACS number(s): 05.70.Fh, 05.70.Ln, 11.30.Pb, 64.70.Kb

\section{INTRODUCTION}

According to the Landau concept a transformation of nonequilibrium thermodynamic system is unequivocally represented by the order parameter (OP). Within the framework of the microscopic theory of phase transitions the appearance of an ordered state is caused by spontaneous symmetry breaking, resulting in switching on a self-consistent field conjugated by the order parameter. At development of the formal scheme of phase transitions, where the values of the order parameter and the self-consistent field change in a self-consistent manner (at a stationary state they are connected by a functional relation), the natural question appeared - will they form a unique mathematical structure type of the vector in a functional space? A positive answer to this question is given by the field scheme developed below, within the framework of which the order parameter and self-consistent field will form a unique superfield, the first component of which is represented by usual (real or complex) values, and the second by the Grassmannian ones.

From the very beginning it is necessary to note, that the term "a superfield" is used by use in the conditional meaning. This field unites usual and Grassmannian variables, instead of various statistics. Within the framework of the initial concept [1] a true superfield has got not two but four components, two of which have Bose character, and two others - the Fermi one. Research of fourcomponents representation has shown [2], that Bose components correspond to the condensate and fluctuation components of the order parameter, and Grassmannian conjugated pair of Fermi components is related to the antiphase boundaries. Obviously, in spatially homogeneous systems, where such boundaries are absent, fourcomponents representation of the superfield become surplus and, in essence, it is reduced to the two-components superfield. Respectively the pair of the Grassmannian conjugated coordinates $\chi, \bar{\chi}$ is reduced to sole $\chi$, and the field scheme should be primarily developed.

Apart from the development of the appropriate formalism, the purpose of the offered work is in the determination of the physical meaning of the two-component superfield. As far as it has Bose character, then as was already mentioned above (see also [2]), its usual component $\eta$ is reduced to the condensate component of the order parameter, and the Grassmannian one $\varphi$ - to the fluctuation constituent. The correlator of these components gives the usual Green function $G(\mathbf{r}, t)=\langle\eta(\mathbf{r}, t) \varphi(\mathbf{0}, 0)\rangle$. However, except the field $\varphi$ it is possible to enter other field $\phi=\varphi-\dot{\eta} / 2 \theta$, where the point means time derivative, $\theta$ - degree of system nonequilibrium. Taking into account, that the value $2 \theta \varphi$ represents an amplitude of the fluctuation component [2], it is possible to show, that the new field $\phi$ is reduced to the deterministic component of the conjugated field. Further, this derivation will be confirmed by the direct calculations. Meaning in mind the possible applications of the developed formalism, we shall consider both the real fields $\eta, \phi, \varphi$, and the complex one (see sections 2 and 3 respectively). In the first case for $\Phi^{4}$-model, taking into account the interaction and the first order power in unharmonicity series it is possible the complete consideration. For the complex fields the calculation becoms much complicated and the final results are reached only numerically.

\section{THE PROBLEM FORMULATION}

We shall proceed from the Langevin equation

$$
\dot{\eta}(\mathbf{r}, t)=-\frac{\partial V}{\partial \eta^{*}}+\zeta(\mathbf{r}, t),
$$

which determining time-spatial dependence of the nonconserved complex order parameter $\eta(\mathbf{r}, t)$. Here the 
point means time derivative, $V(\eta)$ - synergetic potential, $\zeta(\mathbf{r}, t)$ - stochastic component, normalized by conditions of white noise

$$
\langle\zeta(\mathbf{r}, t)\rangle=0, \quad\left\langle\zeta^{*}(\mathbf{r}, t) \zeta(\mathbf{0}, 0)\right\rangle=2 \theta \delta(\mathbf{r}) \delta(t)
$$

where angular brackets mean averaging, $\theta$ - the noise intensity. In the right-hand part (1) before the first term the operator $-\nabla^{2}$ and the operator $-(\mathrm{D} / \theta) \nabla^{2}$ before $\theta$ in (2) appear for the case of conserved order parameter, where D is diffusion coefficient [2].

The basis for construction of the field scheme is a generating functional $[1-3]$

$$
\begin{aligned}
& Z\{u(\mathbf{r}, t)\}=\int Z\{\eta(\mathbf{r}, t)\} \exp \left(\frac{1}{2} \int\left(u^{*} \eta+u \eta^{*}\right) \mathrm{d} \mathbf{r} \mathrm{d} t\right) \mathrm{D} \eta \\
& Z\{\eta(\mathbf{r}, t)\}=\left\langle\prod_{(\mathbf{r}, t)} \delta\left\{\dot{\eta}(\mathbf{r}, t)+\frac{\partial V}{\partial \eta^{*}}-\zeta(\mathbf{r}, t)\right\} \operatorname{det}\left|\frac{\delta \zeta}{\delta \eta}\right|\right\rangle_{\zeta}
\end{aligned}
$$

Its variation over the test field $u(\mathbf{r}, t)$ gives the observed correlators. $Z\{u\}$ represents functional Laplace transformation of the dependence $Z\{\eta\}$, in which the argument of the $\delta$-function is equation (1). The determinant in (4) in the Ito convention is reduced to the noise intensity $\theta$, providing transition from continuous integration over $\zeta$ to $\eta$. Averaging over $\zeta$ is performed according to the distribution

$$
P\{\zeta\} \propto \exp \left\{-\frac{1}{4 \theta} \int|\zeta(\mathbf{r}, t)|^{2} \mathrm{~d} \mathbf{r} \mathrm{d} t\right\}
$$

following from (2). Using the integral representation

$$
\delta\{x(\mathbf{r}, t)\}=\int_{-i \infty}^{i \infty} \exp \left(-\int x \varphi^{*} \mathrm{~d} \mathbf{r} \mathrm{d} t\right) \mathrm{D} \varphi
$$

and performing averaging over (5), the functional (4) takes the standard form

$$
Z\{\eta(\mathbf{r}, t)\}=\int \exp [-S\{\eta(\mathbf{r}, t), \varphi(\mathbf{r}, t)\}] \mathrm{D} \varphi,
$$

where action $S=\int \mathcal{L} \mathrm{d} \mathbf{r} \mathrm{d} t$ is determined by Lagrangian

$$
\mathcal{L}=\varphi^{*}(\dot{\eta}-\varphi)+\varphi^{*} \frac{\partial V}{\partial \eta^{*}}
$$

Here and below, if the otherwise is not meant, the coordinate $\mathbf{r}$ is measured in units of correlation length $\xi$, the time $t$ - in units of $\theta^{-1}$, and the synergetic potential $V$ - in the scale $\theta$.

Let us enter the field $\phi$, determined by the equality

$$
\dot{\eta}=2 \phi+2 \varphi
$$

Then Lagrangian (8) takes the form

$$
\begin{aligned}
\mathcal{L} & =\frac{|\dot{\eta}|^{2}}{4}-|\phi|^{2}-\frac{1}{2}\left(\phi^{*} \dot{\eta}-\dot{\eta}^{*} \phi\right) \\
& -\phi^{*} \frac{\partial V}{\partial \eta^{*}}+\frac{\dot{\eta}^{*}}{2} \frac{\partial V}{\partial \eta^{*}}
\end{aligned}
$$

After substituting into (7) the last term, which represents a complete derivative of $V(\eta)$ with respect to $t$, in functional (3) the multiplier appears

$$
\begin{aligned}
\Delta Z & =\int \exp \left\{-\frac{1}{2} \int\left[V\left(\eta_{f}\right)\right.\right. \\
& \left.\left.-V\left(\eta_{i}\right)\right] \mathrm{d} \mathbf{r} \mathrm{d} t\right\} \mathrm{D} \varphi \mathrm{D} \eta_{i} \mathrm{D} \eta_{f},
\end{aligned}
$$

which is determined by the initial $\eta_{i}(\mathbf{r}, t)$ and final $\eta_{f}(\mathbf{r}, t)$ fields of the order parameter. This multiplier describes system relaxation from the state $\eta_{i}$ to $\eta_{f}$. After its separation it is possible to omit the last term in (10). For representing the rest of the terms into a canonical form let us introduce the superfield

$$
\Phi=\eta+\chi \phi
$$

where Grassmannian coordinate $\chi$ has the usual properties

$$
\chi \chi^{\prime}+\chi^{\prime} \chi=0, \quad \int \mathrm{d} \chi=0, \quad \int \chi \mathrm{d} \chi=1 .
$$

As a result we obtain Lagrangian

$$
\mathcal{L}=\int\left[\frac{1}{2}\left(\Phi^{*} \overline{\mathrm{D}} \Phi+\Phi \mathrm{D} \Phi^{*}\right)+\mathrm{V}(\Phi)\right] \mathrm{d} \chi
$$


where the superderivative operators are entered in the following form

$$
\begin{array}{ll}
\mathrm{D}=\mathrm{D}_{0}+\mathrm{D}_{1}, & \overline{\mathrm{D}}=\mathrm{D}_{0}-\mathrm{D}_{1} ; \\
\mathrm{D}_{0}=-\left(\frac{\partial}{\partial \chi}+\frac{\chi}{4} \frac{\partial^{2}}{\partial t^{2}}\right), & \mathrm{D}_{1}=\left(1-\chi \frac{\partial}{\partial \chi}\right) \frac{\partial}{\partial t} .
\end{array}
$$

Component $\mathrm{D}_{0}$ contains even order of the time derivative, $\mathrm{D}_{1}$ - only odd order (the first). The equivalence of the last term in (14) and the last member in (10) it is possible is attained by performing a formal expand of the dependence $V(\Phi)$ in Maclaurin's series and getting rid of the supercoordinate $\chi$ in degrees of superfield (12) with the help of equations (13). As to the rest of the terms, their identity follows immediately after substituting (15) into (14). Substituting the superfield (12) into operators (15), it is easy to see that they have the following properties:

$$
\begin{aligned}
& \mathrm{D}_{0}^{2 n} \Phi=2^{-2 n} \frac{\partial^{2 n}}{\partial t^{2 n}} \Phi, \\
& \mathrm{D}_{1}^{n} \Phi=\frac{\partial^{n}}{\partial t^{n}} \eta, \quad n=1,2, \ldots
\end{aligned}
$$

On the other hand, the action of the infinitesimal operators $\mathrm{e}^{\varepsilon \mathrm{D}}, \mathrm{e}^{\varepsilon \overline{\mathrm{D}}}, \varepsilon \rightarrow 0$ gives

$$
\begin{aligned}
& \mathrm{e}^{\varepsilon \mathrm{D}}: \chi \rightarrow \chi-\varepsilon ; \quad t \rightarrow t+\varepsilon ; \\
& \mathrm{e}^{\varepsilon \overline{\mathrm{D}}}: \chi \rightarrow \chi-\varepsilon ; \quad t \rightarrow t-\varepsilon . \\
& \mathrm{e}^{\varepsilon \mathrm{D}}: \delta \eta=\varepsilon(\dot{\eta}-\phi) ; \quad \delta \phi=-\frac{\varepsilon}{4} \ddot{\eta} ; \\
& \mathrm{e}^{\varepsilon \overline{\mathrm{D}}}: \delta \eta^{*}=-\varepsilon\left(\dot{\eta}^{*}+\phi^{*}\right) ; \quad \delta \phi^{*}=-\frac{\varepsilon}{4} \ddot{\eta}^{*} .
\end{aligned}
$$

Thus, the operator D increases time, and $\bar{D}$ reduces it, whereas they both reduce supercoordinate. According to (17) the action of the operators $e^{\varepsilon \mathrm{D}}, e^{\varepsilon \overline{\mathrm{D}}}$ on the component $\eta$ leads to a change along the other one $\phi$, included in the combination with the rate of change of the order parameter $\dot{\eta}$. As to the action on the component $\phi$, it is bound up with the second derivative $\ddot{\eta}$ of time dependence of the order parameter.

For further problem solution it is necessary to substitute Lagrangian (14) into the corresponding Euler equation. The expressions which will be obtained below is substantially dependent on the complex properties of the order parameter and the conjugated field. Let us start with the more simple case.

\section{REAL FIELDS}

Here $\Phi=\Phi^{*}$ and the first term in integrand of Eq.(14) takes the form of $\Phi \mathrm{D}_{0} \Phi$. Correspondingly, the motion equation reads

$$
\mathrm{D}_{0} \Phi=\frac{1}{2} V^{\prime}(\Phi),
$$

where a prime means (variational) derivative on the corresponding argument. Substituting to expressions (12), (15), with the use of Eq.(13) for a component of the superfield we obtain

$$
\begin{aligned}
& \ddot{\eta}=-2 V^{\prime \prime}(\eta) \phi, \\
& 2 \phi=-V^{\prime}(\eta) .
\end{aligned}
$$

Taking into account the definition of the conjugated field $f=-V^{\prime}(\eta)$, one can see, that the component of superfield (12), corresponding to the Grassmann variable, is reduced to the conjugated field: $\phi=\frac{1}{2} f$. Using this condition in definition (9) of the fields $\phi$, it is easy to notice, that Eq.(9) plays a role of the averaged Langevin equation (1).

A system of equations (19a), (19b) allows to find components of the superfield $\eta, \phi$ if the synergetic potential $V(\eta)$ is known. In this way asymmetry of equations (19a), (19b) with respect to the time derivatives order is apparent. It vanishes, with the transition from the conjugated field to the fluctuation field $\varphi$ with the help of relation (9). The corresponding equations take the form of

$$
\begin{aligned}
& \dot{\eta}=-V^{\prime}(\eta)+2 \varphi \\
& \dot{\varphi}=V^{\prime \prime}(\eta) \varphi
\end{aligned}
$$

For further progress of the problem we shall pick out interaction in the synergetic potential:

$$
\begin{aligned}
& V\{\Phi\}=V_{0}\{\Phi\}+V_{\mathrm{int}}\{\Phi\}, \\
& V_{0}\{\Phi\}=\int\left[\frac{1}{2 \tau} \Phi^{2}+\frac{1}{2}(\nabla \Phi)^{2}\right] \mathrm{d} \mathbf{r} \mathrm{d} \chi, \\
& V_{\mathrm{int}}\{\Phi\}=\iint V_{\mathrm{int}}\left\{\chi, \chi^{\prime}\right\} \mathrm{d} \chi \mathrm{d} \chi^{\prime}, \\
& V_{\mathrm{int}}\left\{\chi, \chi^{\prime}\right\}=\frac{1}{2} \iint \Phi\left(\mathbf{r}^{\prime}, t^{\prime}, \chi^{\prime}\right) \Phi(\mathbf{r}, t, \chi) \\
& \times v\left(\mathbf{r}, \mathbf{r}^{\prime}\right) \Phi(\mathbf{r}, t, \chi) \Phi\left(\mathbf{r}^{\prime}, t^{\prime}, \chi^{\prime}\right) \mathrm{d} \mathbf{r} \mathrm{d} \mathbf{r}^{\prime}, \\
& v\left(\mathbf{r}, \mathbf{r}^{\prime}\right)=v(\mathbf{r}) \delta\left(\mathbf{r}-\mathbf{r}^{\prime}\right)-w\left(\mathbf{r}-\mathbf{r}^{\prime}\right) .
\end{aligned}
$$

Here in kernel $v\left(\mathbf{r}, \mathbf{r}^{\prime}\right)$ the contributions are divided, caused by self-action (unharmonicity) of $v(\mathbf{r})$ and the two-particle interaction $w\left(\mathbf{r}-\mathbf{r}^{\prime}\right)$, the sign of which specifies the attractive character. In accordance with (21) the nonlinear component has the form of 


\section{REPRESENTATION OF NONEQUILIBRIUM THERMODYNAMIC SYSTEM...}

$$
V_{\mathrm{int}}\left(\chi, \chi^{\prime}\right)=U\left(\chi, \chi^{\prime}\right)-W\left(\chi, \chi^{\prime}\right)
$$

In the first term, representing self-action, it is natural to put $t=t^{\prime}, \chi=\chi^{\prime}$. As a result we obtain the expression

$$
U\left(\chi, \chi^{\prime}\right)=\frac{1}{2} \delta\left(t-t^{\prime}\right)\left(\chi-\chi^{\prime}\right) \int v(\mathbf{r}) \Phi^{4}(\mathbf{r}, t, \chi) \mathrm{d} \mathbf{r},
$$

where the multiplier $\chi-\chi^{\prime}$ corresponds, in accordance with (13), to the Grassmannian $\delta$-function. Below the supersymmetric perturbation theory will be constructed on the basis of this expression. Obviously, by this it is not necessary to use coordinate representation. On the contrary, it is convenient to use the spatial Fourier transform in the second term of (22), which represents a two-particle interaction. As a result it takes the form of

$$
W\left(\chi, \chi^{\prime}\right)=\frac{1}{2} \sum_{\mathbf{k}, \mathbf{k}^{\prime}, \mathbf{q}} \Phi_{-\mathbf{k}+\mathbf{q}}\left(t^{\prime}, \chi^{\prime}\right) \Phi_{\mathbf{k}}(t, \chi) w_{\mathbf{k}-\mathbf{k}^{\prime}} \Phi_{-\mathbf{k}^{\prime}}(t, \chi) \Phi_{\mathbf{k}^{\prime}-\mathbf{q}}\left(t^{\prime}, \chi^{\prime}\right) .
$$

In the mean-field approximation the sum over $\mathbf{q}$ is reduced to sole term $\mathbf{q}=0$, and the pair of multipliers, corresponding to the various arguments, is replaced by average

$$
\left\langle C_{\mathbf{k}}\left(t, \chi ; t^{\prime}, \chi^{\prime}\right)\right\rangle=\left\langle\Phi_{-\mathbf{k}}(t, \chi) \Phi_{\mathbf{k}}\left(t^{\prime}, \chi^{\prime}\right)\right\rangle .
$$

Performing in (24) designate $\mathbf{k}^{\prime}$ on $-\mathbf{k}^{\prime}$, for second term (22) we obtain

$$
\begin{aligned}
& W\left(t, \chi ; t^{\prime}, \chi^{\prime}\right)=\frac{1}{2} \sum_{\mathbf{k}, \mathbf{k}^{\prime}} \Phi_{-\mathbf{k}}(t, \chi) \Phi_{\mathbf{k}}\left(t^{\prime}, \chi^{\prime}\right) w_{\mathbf{k}, \mathbf{k}^{\prime}} C_{\mathbf{k}^{\prime}}\left(t-t^{\prime}, \chi-\chi^{\prime}\right), \\
& w_{\mathbf{k}, \mathbf{k}^{\prime}} \equiv w_{\mathbf{k}+\mathbf{k}^{\prime}}+w_{2 \mathbf{k}} \delta_{\mathbf{k}, \mathbf{k}^{\prime}}
\end{aligned}
$$

As will be shown below, the supercorrelator (25) gives the basic observed values - the structural factor $\langle\eta(\mathrm{r}, t) \eta(0,0)\rangle$ and susceptibility $\langle\eta(\mathrm{r}, t) \varphi(0, t)\rangle$. Therefore it is necessary to pass from the motion equation (18) for the superfield $\Phi_{\mathbf{k}}$ to the appropriate equation for the supercorrelator $C_{\mathbf{k}}\left(z, z^{\prime}\right)$, where $z$ designates a set of values $t, \chi$. With this purpose we shall pick out bare component $C_{\mathbf{k}}^{(0)}\left(z, z^{\prime}\right)$, which obeys the equation

$$
\begin{aligned}
& L_{\mathbf{k}}(z) C_{\mathbf{k}}^{(0)}\left(z, z^{\prime}\right)=\delta\left(z-z^{\prime}\right) \\
& L_{\mathbf{k}}(z) \equiv \mathrm{D}_{0}-\left(2 \tau_{\mathbf{k}}\right)^{-1}, \\
& \delta(z) \equiv \chi \delta(t), \quad \tau_{\mathbf{k}} \equiv \tau\left(1+\mathbf{k}^{2}\right)^{-1} .
\end{aligned}
$$

After time Fourier transformation its solution takes the form of

$$
C_{\omega \mathbf{k}}^{(0)}=\frac{-2 \tau_{\mathbf{k}}^{-1}\left(\chi-\chi^{\prime}\right)+4+\chi \chi^{\prime} \omega^{2}}{\omega^{2}+\tau_{\mathbf{k}}^{-2}}
$$

Peculiarity of this expression consists in the presence of terms containing multipliers $\chi^{0}, \chi, \chi^{\prime}, \chi \chi^{\prime}$. Taking into account properties (13) it is easy to see, that such structure is inherent of not only bare, but also of all super- correlators.

Therefore it is convenient to introduce basic supercorrelators $\mathbf{B}_{ \pm}, \mathbf{T}_{ \pm}$, which is determined by equalities

$$
\mathbf{B}_{ \pm}\left(\chi, \chi^{\prime}\right)=\chi \pm \chi^{\prime}, \quad \mathbf{T}_{ \pm}\left(\chi, \chi^{\prime}\right)=1 \pm \chi \chi^{\prime}
$$

Using the multiplication rule

$$
A\left(\chi, \chi^{\prime}\right)=\int B\left(\chi, \chi^{\prime \prime}\right) C\left(\chi^{\prime \prime}, \chi^{\prime}\right) \mathrm{d} \chi^{\prime \prime}
$$

it is easy to see, that operators (29) obey the multiplication table: 
On the other hand, representing superfield (12) as a vector-column with components $\eta$, $\phi$, we obtain the matrix representation [5]

$$
\begin{aligned}
& \left\|\mathbf{B}_{ \pm}\right\|=\left(\begin{array}{cc} 
\pm 1 & 0 \\
0 & 1
\end{array}\right) \\
& \left\|\mathbf{T}_{ \pm}\right\|=\left(\begin{array}{cc}
0 & 1 \\
\pm 1 & 0
\end{array}\right) \\
& |\Phi|=\left(\begin{array}{l}
\eta \\
\phi
\end{array}\right)
\end{aligned}
$$

The relations obtained above show that the operators $\mathbf{B}_{ \pm}, \mathbf{T}_{ \pm}$will form complete basis for the decomposition of the supercorrelators. So, expression (28) is written down as

$$
\begin{aligned}
& \mathbf{C}^{(0)}=g_{0}^{(0)} \mathbf{B}_{+}+g_{1}^{(0)} \mathbf{B}_{-}+S_{0}^{(0)} \underline{f} T_{+}+S_{1}^{(0)} \mathbf{T}_{-}, \\
& g_{0}^{(0)}=0, \quad g_{1}^{(0)}=-\frac{2 \tau}{1+(\omega \tau)^{2}} \\
& S_{0,1}^{(0)}=\frac{1}{2} \frac{(2 \tau)^{2} \pm(\omega \tau)^{2}}{1+(\omega \tau)^{2}}
\end{aligned}
$$

where for the sake of brevity the indeces $\omega, \mathbf{k}$ are omitted. Substituting superfield (12) into definition (25), with the account of (29) one can see, that the components $g_{ \pm}=g_{0} \pm g_{1}, S_{ \pm}=S_{0} \pm S_{1}$ give the observed correlators:

$$
\begin{array}{ll}
\langle\eta \eta\rangle=S_{+}, \quad\langle\eta \phi\rangle=g_{-}, \\
\langle\phi \eta\rangle=g_{+}, \quad\langle\phi \phi\rangle=S_{-} .
\end{array}
$$

In accordance with (32) in linear approximation they have a standard form

$$
g_{-}^{(0)}=-g_{+}^{(0)}=\frac{2 \tau}{1+(\omega \tau)^{2}}
$$

$$
S_{+}^{(0)}=\frac{(2 \tau)^{2}}{1+(\omega \tau)^{2}}, \quad S_{-}^{(0)}=\frac{(\omega \tau)^{2}}{1+(\omega \tau)^{2}}
$$

Taking into account (9) from Eqs.(34) we obtain usual expression for response function $\langle\eta \varphi\rangle=2 \tau(1+i \omega \tau)^{-1}$. The relation $S_{+}^{(0)}=2 \tau g_{-}$corresponds to the fluctuationdissipation theorem, and decreasing the autocorrelator $S_{-}^{(0)} \propto \omega^{2}$ in a hydrodynamic limit $\omega \rightarrow 0$ justifies the separation conjugated field. Using Fourier-images for the moment of time $t=0$ we obtain

$$
\begin{aligned}
& S_{+}^{(0)}(0)=2 \tau, \quad g_{ \pm}^{(0)}(0)=\mp 1 \\
& S_{-}^{(0)}(0)=(2 \tau)^{-1}(C-1)
\end{aligned}
$$

where term $C \rightarrow \infty$ corresponds to ultra-violet divergence of the autocorrelator of the conjugated field at $t \rightarrow 0$. Obviously, the given divergence is stipulated by the peculiarity of the mean field approximation and really $C<\infty$. Further the magnitude $C$ will be obtained according to physical reasons.

For the transition from bare correlators to the exact ones it is necessary to take into account interaction (22) which is written down in approximation to the selfconsistent field. In order to prevent nonlinear terms in expression for $W\{\Phi\}$ we accept $\mathbf{C} \simeq \mathbf{C}^{(0)}$. As a result, the Dyson equation is written down as

$$
\mathbf{C}^{-1}=\left(\mathbf{C}^{(0)}\right)^{-1}-\mathbf{\Sigma}-w \mathbf{C}^{(0)}
$$

where $\boldsymbol{\Sigma}$ means the self-energy function. Carrying out the decomposition type of (32) for the $\boldsymbol{\Sigma}, \mathbf{C}$

$$
\begin{aligned}
& \boldsymbol{\Sigma}=\sigma_{0} \mathbf{B}_{+}+\sigma_{1} \mathbf{B}_{-}+\Sigma_{0} \mathbf{T}_{+}+\Sigma_{1} \mathbf{T}_{-}, \\
& \mathbf{C}=g_{0} \mathbf{B}_{+}+g_{1} \mathbf{B}_{-}+S_{0} \mathbf{T}_{+}+S_{1} \mathbf{T}_{-},
\end{aligned}
$$

after the substitution in (36) we obtain

$$
\begin{aligned}
& \mathcal{D} g_{ \pm}=g_{ \pm}^{(0)}+\left(\sigma_{ \pm}+w g_{ \pm}^{(0)}\right) \Delta \\
& \mathcal{D} S_{ \pm}=S_{ \pm}^{(0)}-\left(\Sigma_{ \pm}+w S_{ \pm}^{(0)}\right) \Delta \\
& \mathcal{D} \equiv 1-\left[\left(\sigma_{+} g_{+}^{(0)}+\sigma_{-} g_{-}^{(0)}\right)+\left(\Sigma_{+} S_{-}^{(0)}+\Sigma_{-} S_{+}^{(0)}\right)\right] \\
& +\Delta\left(\Sigma_{+} \Sigma_{-}-\sigma_{+} \sigma_{-}\right)-w\left\{\left(g_{+}^{(0)} g_{+}^{(0)}+g_{-}^{(0)} g_{-}^{(0)}+2 S_{+}^{(0)} S_{-}^{(0)}\right)\right. \\
& \left.-\Delta\left[\left(\Sigma_{+} S_{-}^{(0)}+\Sigma_{-} S_{+}^{(0)}\right)-\left(\sigma_{+} g_{-}^{(0)}+\sigma_{-} g_{+}^{(0)}\right)\right]\right\}+w^{2} \Delta^{2} \\
& \Delta \equiv S_{+}^{(0)} S_{-}^{(0)}-g_{+}^{(0)} g_{-}^{(0)} .
\end{aligned}
$$


Here bare components $g_{ \pm}^{(0)}, S_{+}^{(0)}$ are given by expressions (34).

So as to find the components $\sigma_{0,1}, \Sigma_{0,1}$, which determine self-energy function (37), let us use the first order of the perturbation theory over unharmonicity (23). With this purpose it is necessary to find second derivative of the functional $Z\{\Phi(\mathbf{r}, t, \chi)\}$ type of $(7)$ over superfield $\Phi(\mathbf{r}, t, \chi)$. Then there will by a carry out decomposition of $Z\{\Phi(\mathbf{r}, t, \chi)\}$ over the components of the actions corresponding to contribution (23). Accepting the kernel $v(\mathbf{r})$ to be independent on the coordinate, we obtain the first correction to the supercorrelator(25) as

$$
\mathbf{C}^{(1)}\left(z, z^{\prime}\right)=-\frac{v}{4}\left\langle\Phi(z) \int \Phi^{4}\left(z_{1}\right) \mathrm{d} z_{1} \Phi\left(z^{\prime}\right)\right\rangle_{0},
$$

where $z$ is a set of the values $\mathbf{r}, t, \chi$, and averaging will be carried out over a free field. It is necessary to use the Wick theorem, representing average over six multipliers $\Phi$ by product of the three bare supercorrelators type of (25). Taking into account, that the number of possible coupling to such supercorrelators resulting in (39) equals 12 , we obtain

$$
\mathbf{C}^{(1)}\left(z, z^{\prime}\right)=\int \mathbf{C}^{(0)}\left(z, z_{1}\right) \mathbf{\Sigma}\left(z-z^{\prime}\right) \mathbf{C}^{(0)}\left(z_{1}, z^{\prime}\right) \mathrm{d} z_{1},
$$

where the self-energy part is determined by equality

$$
\boldsymbol{\Sigma}\left(z-z^{\prime}\right)=-3 v \delta\left(z-z^{\prime}\right) \mathbf{C}^{(0)}\left(z_{1}, z_{1}\right)
$$

Using the definition of basic correlators (29), we come to the expression

$$
\boldsymbol{\Sigma}(t)=-3 v \mathbf{B} \_\mathbf{C}^{(0)}(t=0) \delta(t) .
$$

Substituting here Fourier-image of decomposition (32), in accordance with (35) and the table of multiplication of basic supercorrelators we obtain

$$
\begin{aligned}
& \sigma_{0}=-3 v g_{0}^{(0)}(0) \delta(t)=0 \\
& \sigma_{1}=-3 v g_{1}^{(0)}(0) \delta(t)=3 v \delta(t) \\
& \Sigma_{0,1}=3 v S_{0,1}^{(0)}(0) \delta(t)=3 v \tau\left[1 \pm(2 \tau)^{-2}(C-1)\right] \delta(t) .
\end{aligned}
$$

The use of expressions (34), (43) in equalities (38) gives the following result for correlators $(33)$ :

$$
\begin{aligned}
& \mathcal{D} g_{ \pm}=\mp \frac{2 \tau}{1+(\omega \tau)^{2}}\left[(1-6 v \tau)-\frac{4 w \tau^{2}}{1+(\omega \tau)^{2}}\right] \\
& \mathcal{D} S_{+}=\frac{(2 \tau)^{2}}{1+(\omega \tau)^{2}}\left[(1-6 v \tau)-\frac{4 w \tau^{2}}{1+(\omega \tau)^{2}}\right]
\end{aligned}
$$

$$
\begin{aligned}
& \mathcal{D} S_{-}=\frac{(\omega \tau)^{2}}{1+(\omega \tau)^{2}} \\
& -\frac{(2 \tau)^{2}}{1+(\omega \tau)^{2}}\left[\frac{3 v}{2 \tau}(C-1)+w \frac{(\omega \tau)^{2}}{1+(\omega \tau)^{2}}\right]
\end{aligned}
$$

The hydrodynamic limit $\omega \rightarrow 0$ should give condition $S_{-} \rightarrow 0$, therefore in the last expressions in the (44) it is necessary to put $C=1$. As a result the autocorrelator of the conjugated field takes the form of

$$
\mathcal{D} S_{-}=\frac{(\omega \tau)^{2}}{1+(\omega \tau)^{2}}\left(1-\frac{4 w \tau^{2}}{1+(\omega \tau)^{2}}\right) .
$$

Accordingly, for the denominator $\mathcal{D}$ in linear approximation over $v$ we find

$$
\begin{aligned}
& \mathcal{D} \simeq(1-6 v \tau)-\frac{8 w \tau^{2}-6 v \tau\left(3+4 w \tau^{2}\right)}{1+(\omega \tau)^{2}} \\
& +\frac{8 w \tau^{3}(2 w \tau-9 v)}{\left[1+(\omega \tau)^{2}\right]^{2}} .
\end{aligned}
$$

The condition $\mathcal{D}=0$ results in the dispersion law $\omega=-i \tau_{\mathrm{ef}}^{-1}$, from which the effective relaxation time is determined as follows:

$$
\tau_{\mathrm{ef}}=\tau\left(\frac{1-6 v \tau}{1+12 v \tau-4 w \tau^{2}}\right)^{\frac{1}{2}}
$$

From here it is visible, that at $4 w \tau^{2}-12 v \tau=1$ we have $\tau_{\text {ef }}=\infty$, and the system gets instable, which corresponds to phase transition to the order state. Returning to the measure units and taking into account a Cure ratio $\tau=(2 \theta)^{-1}$, we find a point of stability breaking

$$
\theta_{c}=w-6 v
$$

Thus, in agreement with the usual theory of phase transitions (see [4]) the two-particle interaction promotes system ordering, and prevents unharmonicity.

\section{COMPLEX FIELDS}

Varying the action corresponding to Lagrangian (14) over $\Phi^{*}, \Phi$ we obtain the motion equations for the complex conjugated fields:

$$
\begin{aligned}
& L_{00} \Phi\left(z^{\prime}\right)=2 \frac{\delta V_{\mathrm{int}}}{\delta \Phi^{*}\left(z^{\prime}\right)}, \quad L_{00} \equiv \mathrm{D}_{1}-(2 \tau)^{-1} \\
& L_{11} \Phi^{*}\left(z^{\prime}\right)=2 \frac{\delta V_{\mathrm{int}}}{\delta \Phi\left(z^{\prime}\right)}, \quad L_{11} \equiv-\mathrm{D}_{1}-(2 \tau)^{-1} .
\end{aligned}
$$


It is significant that they differ in sign of the operator $\mathrm{D}_{1}$ of the first time derivative and do not contain operator $\mathrm{D}_{0}$ of the even degrees $\partial^{n} / \partial t^{n}, n=0,2$. As the latter appeared in the motion equation (18) for the real field it is necessary to complete systems (49a), (49b) by the equations

$$
\begin{array}{ll}
L_{10} \Phi\left(z^{\prime}\right)=2 \frac{\delta V_{\mathrm{int}}}{\delta \Phi^{*}\left(z^{\prime}\right)}, & L_{10}=\mathrm{D}_{0}-(2 \tau)^{-1} \\
L_{01} \Phi^{*}\left(z^{\prime}\right)=2 \frac{\delta V_{\mathrm{int}}}{\delta \Phi\left(z^{\prime}\right)}, & L_{01}=\mathrm{D}_{0}-(2 \tau)^{-1}
\end{array}
$$

In contrast to Eqs.(49a), (49b) here transition to the complex conjugated field, meaning conversion of time, does not change sign before the operator $\mathrm{D}_{0}$.

Let us multiply equations (49a), (50b) by $\Phi(z)$, and (49b), (50a) by $\Phi^{*}(z)$, then substitute there the nonlinear components $(23),(24)$ and carry out averaging of the obtained expressions. Then, splitting nonlinear terms by means of the Wick theorem, we obtain

$$
\begin{aligned}
& L^{\alpha \beta}\left(\chi, \chi^{\prime}\right) C^{\beta \gamma}\left(\chi, \chi^{\prime}\right)=\delta^{\alpha \gamma} \delta\left(\chi, \chi^{\prime}\right) \\
& +w C^{01}(\chi, \chi) C^{\alpha \gamma}\left(\chi, \chi^{\prime}\right)+\Sigma^{\alpha \beta}\left(\chi, \chi^{\prime}\right) C^{\beta \gamma}\left(\chi, \chi^{\prime}\right)
\end{aligned}
$$

Here all the values have got matrix structure, reflected by Greek indeces, accepting values 0, 1 (over the repeated indeces summation is meant). So, the supercorrelator (25) has matrix elements

$$
\begin{aligned}
& C^{00}\left(z, z^{\prime}\right)=\left\langle\Phi(z) \Phi\left(z^{\prime}\right)\right\rangle, \\
& C^{01}\left(z, z^{\prime}\right)=\left\langle\Phi(z) \Phi^{*}\left(z^{\prime}\right)\right\rangle, \\
& C^{10}\left(z, z^{\prime}\right)=\left\langle\Phi^{*}(z) \Phi\left(z^{\prime}\right)\right\rangle, \\
& C^{11}\left(z, z^{\prime}\right)=\left\langle\Phi^{*}(z) \Phi^{*}\left(z^{\prime}\right)\right\rangle,
\end{aligned}
$$

where indeces specify the number of complex conjugated signs at the corresponding multipliers $\Phi$. As well as in case of real order parameter, each of these elements is decomposed over the basic operators $\mathbf{B}_{ \pm}, \mathbf{T}_{ \pm}$. Designating the matrix with elements $(52)$ as $\widehat{\mathbf{C}}$, it is possible to present this decomposition by equality type of (37), where the coefficients $\widehat{g}_{0,1}, \widehat{S}_{0,1}$ will also have matrix structure. Then, after the multiplication of equality (52) by $\left(C^{\gamma \delta}\left(\chi^{\prime}, \chi^{\prime \prime}\right)\right)^{-1}$, its summation over the index $\gamma$ and integration over the argument $\chi^{\prime}$ we obtain the matrix Dyson equation (compare with (36))

$$
\widehat{\mathbf{C}}^{-1}=\left(\widehat{\mathbf{C}}^{(0)}\right)^{-1}-w \widehat{\widetilde{\mathbf{C}}}-\widehat{\Sigma}
$$

Here the supercorrelator $\widehat{\widetilde{\mathbf{C}}}$ has coincident matrix elements type of $\mathbf{C}^{01(0)}(\chi, \chi)$.

To ascertain the physical meaning of the elements of the matrices $\widehat{g}_{0,1}, \widehat{S}_{0,1}$ it is necessary to pass to the corre- lators $\widehat{g}_{ \pm}=\widehat{g}_{0} \pm \widehat{g}_{1}, \widehat{S}_{ \pm}=\widehat{S}_{0} \pm \widehat{S}_{1}$ and to use definitions (33), (52). So, for the matrix $\widehat{S}_{+}$we obtain

$$
\begin{aligned}
& \langle\eta \eta\rangle=S_{+}^{00}, \quad\left\langle\eta \eta^{*}\right\rangle=S_{+}^{01} \\
& \left\langle\eta^{*} \eta\right\rangle=S_{+}^{10}, \quad\left\langle\eta^{*} \eta^{*}\right\rangle=S_{+}^{11} .
\end{aligned}
$$

At definition of the matrix $\widehat{g}_{-}$it is necessary to replace $\eta$ for $\phi$ in the second multiplier, for the matrix $\widehat{g}_{+}$- in the first, and for the autocorrelator of the conjugated field $\widehat{S}_{-}$- in both. Obviously, physically observable values will be only nondiagonal matrix elements.

At first let us determine the bare supercorrelator matrices $\widehat{\mathbf{C}}^{(0)}=\widehat{\mathbf{L}}^{-1}$. Because the elements $L_{01}=L_{10}$ have according to Eqs.(50a), (50b) the same form as for the real order parameter, then the nondiagonal elements of matrices $\widehat{g}_{0,1}, \widehat{S}_{0,1}$ are set by equality (32). For the determination of the diagonal elements it is necessary to convert the operators $L_{00}, L_{11}$ given by equality (49a), (49b). With this purpose let us pick out from the operator $\mathrm{D}_{1}$ term $(1 / 2) \partial / \partial t$ and multiply the numerator and the denominator of expression

$$
L_{00}^{-1}=1 /\left\{\left[-(2 \tau)^{-1}-(1 / 2) \partial / \partial t\right]+\left[\mathrm{D}_{1}(1 / 2) \partial / \partial t\right]\right\}
$$

by a sum of the members, which is in square brackets [6]. As a result, the bare correlator $C_{00}^{(0)}\left(\chi, \chi^{\prime}\right)=L_{00}^{-1}\left(\chi, \chi^{\prime}\right)$ takes the form of

$$
C_{00}^{(0)}\left(\chi, \chi^{\prime}\right)=2 \tau \frac{-\chi(1+2 i \omega \tau)+\chi^{\prime}}{1+2 i \omega \tau}
$$

In a similar way we obtain

$$
C_{11}^{(0)}\left(\chi, \chi^{\prime}\right)=2 \tau \frac{-\chi(1-2 i \omega \tau)+\chi^{\prime}}{1-2 i \omega \tau}
$$

Using definition (29), for a component of decomposition (32) one has

$$
\begin{aligned}
& g_{0}^{(0) 00}=-2 \tau \frac{i \omega \tau}{1+2 i \omega \tau}, \quad g_{1}^{(0) 00}=-2 \tau \frac{1+i \omega \tau}{1+2 i \omega \tau} \\
& g_{0}^{(0) 11}=2 \tau \frac{i \omega \tau}{1-2 i \omega \tau}, \quad g_{1}^{(0) 11}=-2 \tau \frac{1-i \omega \tau}{1-2 i \omega \tau}
\end{aligned}
$$

All other components of these matrices elements equal zero. In agreement with the case $t=0$ time Fourierimages take the form of

$$
\begin{aligned}
& g_{0}^{(0) 00}(0)=-g_{0}^{(0) 11}(0)=1 / 2 \\
& g_{1}^{(0) 00}(0)=g_{1}^{(0) 11}(0)=-1 / 2 .
\end{aligned}
$$

As a result, we find final expressions for matrices of the bare correlators from expressions (34), (56): 


$$
\begin{aligned}
& \widehat{g}_{+}^{(0)}=-2 \tau\left(\begin{array}{cc}
1 & {\left[1+(\omega \tau)^{2}\right]^{-1}} \\
{\left[1+(\omega \tau)^{2}\right]^{-1}} & 1
\end{array}\right), \\
& \widehat{g}_{-}^{(0)}=2 \tau\left(\begin{array}{cc}
(1+2 i \tau \omega)^{-1} & {\left[1+(\omega \tau)^{2}\right]^{-1}} \\
{\left[1+(\omega \tau)^{2}\right]^{-1}} & (1-2 i \tau \omega)^{-1}
\end{array}\right), \\
& \widehat{S}_{+}^{(0)}=\frac{(2 \tau)^{2}}{1+(\omega \tau)^{2}}\left(\begin{array}{ll}
0 & 1 \\
1 & 0
\end{array}\right) \\
& \widehat{S}_{-}^{(0)}=\frac{(\omega \tau)^{2}}{1+(\omega \tau)^{2}}\left(\begin{array}{ll}
0 & 1 \\
1 & 0
\end{array}\right) .
\end{aligned}
$$

The calculation of matrix elements of the self-energy function $\widehat{\boldsymbol{\Sigma}}$ reads (compare with (42))

$$
\begin{aligned}
& \boldsymbol{\Sigma}_{00}(t)=-\frac{1}{2} v \mathbf{B}_{-} \mathbf{C}_{11}^{(0)}(t=0) \delta(t), \\
& \boldsymbol{\Sigma}_{01}(t)=-v \mathbf{B}_{-} \mathbf{C}_{10}^{(0)}(t=0) \delta(t), \\
& \boldsymbol{\Sigma}_{10}(t)=-v \mathbf{B}_{-} \mathbf{C}_{01}^{(0)}(t=0) \delta(t), \\
& \boldsymbol{\Sigma}_{11}(t)=-\frac{1}{2} v \mathbf{B}_{-} \mathbf{C}_{00}^{(0)}(t=0) \delta(t) .
\end{aligned}
$$

Decomposing $\widehat{\mathbf{C}}^{(0)}(t=0)$ in a series as (32) with coefficient (35), (57) and using the multiplication rule for the operators $\mathbf{B}_{ \pm}, \mathbf{T}_{ \pm}$,for matrix decomposition type of (37) we find

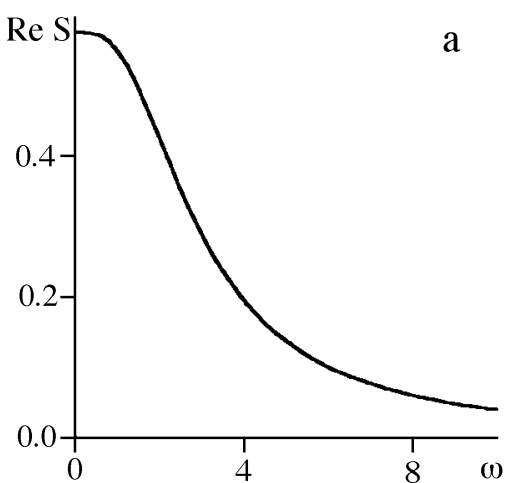

$$
\begin{aligned}
& \widehat{\sigma}_{0}=\frac{v}{4} \delta(t)\left(\begin{array}{cc}
1 & 0 \\
0 & -1
\end{array}\right), \\
& \widehat{\sigma}_{1}=\frac{v}{4} \delta(t)\left(\begin{array}{cc}
-1 & 4 \\
4 & -1
\end{array}\right), \\
& \widehat{\Sigma}_{0,1}=v \tau \delta(t)\left(\begin{array}{ll}
0 & 1 \\
1 & 0
\end{array}\right) .
\end{aligned}
$$

Substituting the expressions (59), (61) to matrix equation (53) and separating result over components, we obtain a system of 16 linearized equations for elements of the exact supercorrelator, which are determined by equalities type of Eqs.(52). Though analytical solution of this system does not lead to question of principle, as a result very cumbersome expressions are obtained, is convenient by presentable in a graphic form. In figure 1 the form of the frequency dependence is shown for the autocorrelator

$$
S=\frac{1}{2}\left(\left\langle\eta \eta^{*}\right\rangle+\left\langle\eta^{*} \eta\right\rangle\right)=\frac{1}{2}\left(S_{+}^{(01)}+S_{+}^{(10)}\right) .
$$

It is obvious, that with raising the particle interaction parameter $w$ this dependence changes the form - its maximum shifts to the point $\omega \neq 0$. In other words, the most preferable state becomes dynamical, in contrast to the usual static one.
[1] G. Parisi, N. Sourlas, Phys. Rev. Lett. 43, 744 (1979).

[2] A. I. Olemskoi, I. V. Koplyk, Usp. Fiz. Nauk 165, No 10, 1105 (1995).

[3] K. Itzikson, J.-B. Zuber, Quantum Field Theory, v. 1, 2 (Translated into Russian) (Mir Publishers, Moskva, 1984).

[4] A. A. Katsnel'son, A. I. Olemskoi, The Microscopic Theory of Nonhomogeneous Structures (Mir Publishers, Moskva, 1990).

[5] Let us specify in order to prevent misunderstanding, that

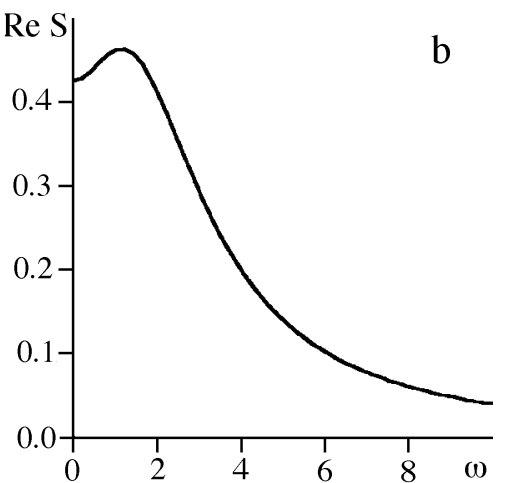

Fig. 1. The frequency dependence of the autocorrelator (62); $\mathrm{a}-\tau=0.5, v=0.1, w=0.2 ; \mathrm{b}-\tau=0.5, v=0.1, w=0.4$. 


\section{ПРЕДСТАВЛЕННЯ НЕРІВНОВАЖНОЇ ТЕРМОДИНАМІЧНОЇ СИСТЕМИ ДВОХКОМПОНЕНТНИМ СУПЕРПОЛЕМ}

О. І. Олємской, І. В. Коплик, В. А. Бражний

Сумсъкий державний університет, Україна, 244007, Суми, вул. Римсъкого-Корсакова, 2

E-mail: postmaster@sgu.sumy.ua

Для термодинамічної системи, що описується рівнянням Ланжевена з білим адитивним шумом, знайдено вираз для твірного функціоналу в залежності від двохкомпонентного суперполя. Звичайна компонента цього поля є параметром впорядкування, а грасманова змінна - самоузгоджене поле, спряжене до цього параметра. В першому порядку за міжатомною взаємодією і ангармонізмом знайдені явні вирази для частотної залежності автокореляторів дійсного параметра впорядкування і відповідного спряженого поля. Визначені умови втрати стійкості і характерний масштаб системи. Для комплексного поля чисельно отриманий вигляд залежності відповідних кореляторів при різних параметрах задачі. 